\title{
Medical Image Segmentation by Level Set Method Incorporating Region and Boundary Statistical Information
}

\author{
Pan Lin, Chongxun Zheng, Yong Yang, and Jianwen Gu \\ Institute of Biomedical Engineering, Key Laboratory of Biomedical Information Engineering \\ of Education Ministry, Xi' an Jiaotong University, xi' an 710049, China \\ Linpan99@sohu.com
}

\begin{abstract}
Level set methods are powerful numerical techniques for image segmentation and analysis. This method requires the definition of a speed function that governs curve evolution. However, the classical method only used image gradient, edge strength, and region intensity to define the speed function. In this paper, we present a new speed function for level set framework. The new method integrates the image region statistical information and image boundary statistical information instead of the conventional method that uses spatial image gradient information. The new speed function gives the level set method a global view of the boundary information within the image. The method here proposed is particularly well adapted to situations where edges are weak and overlap, and images are noisy. A number of experiments on ultrasound, CT, and $\mathrm{X}$-ray modalities medical images were performed to evaluate the new method. The experimental results demonstrate the reliability and efficiency of this new scheme.
\end{abstract}

\section{Introduction}

The level set approach were introduced by S. Osher and J. A. Sethian[1] in 1988. These methods have recently become one of the most studied techniques for medical image segmentation. Level sets are designed to handle problems in which the evolving interfaces can develop sharp corners and cusps, change topology and become very complex. In the level set approach, the convergence to the final result may be relatively independent of the initial shape, and branches, splits and merges can develop without problems as the front moves. Generally, the method may be applied even where no a priori assumptions about the object's topology are made. Most of the challenges in level set approach result from the need to construct an adequate model for the speed function.

But the classical level set speed function models rely on the edge gradient information to stop the curve evolution; these models can detect only objects with edges defined by gradient. Also, in practice, the discrete gradients are bounded and then the stopping function is never zero on the edges, and the curve may pass through the boundary. On the other hand, if the image is very noisy, then the isotropic smooth the edges too. 
To address this problem speed functions that take into account probability density functions of regions inside and outside the structures of interest have been proposed $[3,4,5,6,7]$. This class of solution is well adapted to situations where adjacent structures have different intensity distributions but are challenged by applications that require the segmentation of structures surrounded by other structures with similar intensity values and separated by weak edges.

In this paper, a new speed function model is proposed, which based on the image region and boundary gradient information. The region information is achieved at a global level by a statistical characterization. The boundary finding part is handled by the gradient information. Since the gradient defines a measure of no-homogeneity in the pixel neighborhood, it is modeled as a potential function that generates a Gibbs distribution of a Markov random field [9]. The experimental results show that incorporating region intensity information and gradient information into the level set framework, an accurate and robust segmentation can be achieved.

\section{Level Set Method}

The level set method was proposed by Osher and Sethian[1], The main idea in the level set method is to describe a closed curve $\Gamma$ in the image plane as the zero level set of a higher dimensional function $\phi(x, t)$ in $\mathfrak{R}^{3}$, The value of $\phi$ at some point $\mathrm{x}$ is defined by

$$
\phi(x, t=0)= \pm d
$$

where $d$ is the distance from $\mathrm{x}$ to $\Gamma(t=0)$, and the sign in (1) is chosen whether the point $\mathrm{x}$ lies outside or inside the initial hypersurface. $\Gamma(t=0)$.In this manner, $\Gamma$ is represented by the zero level set $\Gamma(t)=\left\{x \in R^{2} \mid \phi(x, t)=0\right\}$ of the level set function, and the initial function $\phi(x, t=0)$ with the property that $\Gamma(0)=\left\{x \in R^{2} \mid \phi(x, t=0)=0\right\}$. The evolution of $\phi(x, t)$ can be modeled as

$$
\frac{\partial \phi}{\partial t}+F\|\nabla \phi\|=0 \text { With } \phi(x, t=0)
$$

The speed function $F$ plays the major role in the evolution process. The speed function $F$ depends on factors like the image gradient. A common choice for $F$ is:

$$
F=P(I)(1-\varepsilon k)
$$

where, $0<\varepsilon<1$ is a constant, I is the image intensity and $k$ is the curvature obtained from divergence of the gradient of the normal vector to the front. The term $P(I)$ is an image-dependent halting criteria calculated as:

$$
P(I)=e^{-\left|\nabla G_{\sigma} * I\right|}
$$

where $\nabla G * I$ denotes the image convolved with a Gaussian smoothing filter whose characteristic width is $\sigma$. This halting criterion allows model to stop on high image gradient by reducing speed function to zero, thus aligning it to the object boundary. 
Given the initial value, it can be solved by means of difference operators in a fixed grid via

$$
\phi_{i j}{ }^{n+1}=\phi_{i j}{ }^{n}-\Delta t \cdot h \cdot\left(\max \left(F_{i j}, 0\right) \nabla^{+}+\min \left(F_{i j}, 0\right) \nabla^{-}\right)
$$

where $n$ is the iterative time, $h$ is the grid step, $\Delta t$ is the time step, $F_{i j}$ is the speed value of pixel $(i, j), \phi_{i j}{ }^{n}$ is the level value of pixel $(i, j)$ at time $n$ and where

$$
\begin{gathered}
\nabla^{+}=\left[\max \left(D_{i j}{ }^{-x} \phi, 0\right)^{2}+\min \left(D_{i j}{ }^{+x} \phi, 0\right)^{2}+\max \left(D_{i j}{ }^{-y}, 0\right)^{2}+\min \left(D_{i j}{ }^{+y} \phi, 0\right)^{2}\right]^{\frac{1}{2}} \\
\nabla^{-}=\left[\max \left(D_{i j}{ }^{+x} \phi, 0\right)^{2}+\min \left(D_{i j}{ }^{-x} \phi, 0\right)^{2}+\max \left(D_{i j}{ }^{+y}, 0\right)^{2}+\min \left(D_{i j}{ }^{-y} \phi, 0\right)^{2}\right]^{\frac{1}{2}} \\
D_{i j}{ }^{-x} \phi=\frac{\phi_{i j}{ }^{n}-\phi_{i-1 j}{ }^{n}}{\Delta x} D_{i j}{ }^{+x} \phi=\frac{\phi_{i+1 j}{ }^{n}-\phi_{i j}{ }^{n}}{\Delta x} \\
D_{i j}{ }^{-y} \phi=\frac{\phi_{i j}{ }^{n}-\phi_{i j-1}{ }^{n}}{\Delta y} D_{i j}{ }^{+y} \phi=\frac{\phi_{i j+1}{ }^{n}-\phi_{i j}{ }^{n}}{\Delta y}
\end{gathered}
$$

This implementation allows the function $\phi$ to automatically follow topological changes and corners during evolution.

\section{The Proposed Level Set Speed Function}

The key idea was to utilize the probability density function inside and outside the structure to be segmented. The pixel in the neighborhood of the segmenting structure was responsible for creating a pull/push force on the propagating front. The boundary finding part is handled by the gradient information. Since the gradient defines a measure of no-homogeneity in the pixel neighborhood, it is modeled as a potential function that generates a Gibbs distribution of a Markov random field. We chosen the original speed given by Malladi[2] for its simplicity as :

$$
F=h_{I}(v-\rho k)
$$

where $v$ represents an external propagation force, $k$ is the local curvature of the front and acts as a regularization term. The weighting $\rho$ expresses the importance given to regularization. The term $h_{I}$ is the data consistency and act as a stopping criterion at the location of the desired boundaries; which is defined according to the intensity of the image data.

\subsection{Image Region Statistical Characterization}

Suppose that a image is partitioned into $N$ pixel, labeled by the integers $1,2, \cdots, N$. In most application, the pixel locations or sites will form a regular square lattice. Further suppose that each pixel variable, $x_{i}, 1 \leq i \leq N$ can take any real value, $x_{i} \in R$. The values of the pixel variables are called intensities and arbitrary shading will be de- 
noted $x=\left\{x_{1}, x_{2}, \cdots, x_{N}\right\}$, hence $x \in R^{N}$. In general it is not possible to observe $x$ directly, instead the observed image $y$ is a degraded copy of $x$.

$$
y_{i}=x_{i}+\varepsilon_{i} \quad, \quad 1 \leq i \leq N
$$

where $\varepsilon_{i} \sim N\left(0, \sigma^{2}\right)$, and $\varepsilon_{i}$ and $\varepsilon_{j}$ are independent when $i \neq j$.Hence the conditional distribution of $Y$ given $X$ and $\sigma$ with density function:

$$
P(Y \mid X)=\frac{1}{\sqrt{2 \pi} \sigma} \exp \left\{-\frac{1}{2 \sigma^{2}} \sum_{i=1}^{N}\left(y_{i}-x_{i}\right)^{2}\right\}
$$

The sign of $v$ determines the direction of the external propagation force.

$$
\begin{gathered}
v=\operatorname{Sign}\left\{a_{i} p_{i}(I)-\left(1-a_{i}\right) p_{e}(I)\right\} \\
p\left(y_{s} \mid x_{s}\right) \propto \begin{cases}\frac{1}{\sqrt{2 \pi} \sigma_{\text {in }}} \exp \left(-\frac{\left(I(x)-\mu_{\text {in }}\right)^{2}}{2 \sigma^{2}{ }_{\text {in }}}\right) & \text { insideobject } \\
\frac{1}{\sqrt{2 \pi} \sigma_{\text {out }}} \exp \left(-\frac{\left(I(x)-\mu_{\text {out }}\right)^{2}}{2 \sigma_{\text {out }}^{2}}\right) & \text { outsideobject }\end{cases}
\end{gathered}
$$

where $p_{i}(I)$ and $p_{e}(I)$ denote the likehood of intensity inside and outside the object, and $a_{i}$ is the prior of a image to be inside the object. Where $\mu_{\text {in }}, \mu_{\text {out }}, \sigma^{2}$ in and $\sigma_{\text {out }}^{2}$ are respectively the mean and the variance of the image intensity.

\subsection{Image Boundary Statistical Characterization}

Let $Y$ be the observed image, $X$ the ideal image, and $N$ is an additive Gaussian noise present in the image. That can be described as:

$$
Y=X+N
$$

The gradient image is corrupted by false-edges due to noise. The plausibility of false edges follows a Rayleigh distribution. In order to distinguish real edges from false ones, Voorhees [8] proposed to statistically estimate a threshold that separates these two populations.

We assume the plausibility of the true edges being described by a single distribution. One must normalize the gradient values in order to keep both gradient and statistics measures in the same numerical range. Therefore, we define the normalized gradient at a given position $y_{s}$ as follows

$$
\tilde{g}\left(y_{s}\right)=\frac{g\left(y_{s}\right)}{\mu}
$$

where $\mu$ is the mean of the Rayleigh distribution.

The segmentation field, $x$, has an isotropic nature and its distribution is strictly defined in a local neighborhood. Thereafter, we can use MRF model. Based on the Hammersley-Clifford theorem, the density of $x$ is given by a Gibbs density using the form: 


$$
p(x)=\frac{1}{Z} e^{-\left(\sum_{c} V_{c}(X)\right)}
$$

where $Z$ is a normalization factor, or partitioning function. $V_{c}(x)$ is a sum of functions, one of each pixel in $x$, which describes the interaction of each pixel with its neighbors. The clique potentials $V_{c}$ depend only on the pixels that belong to clique $C$. Since the gradient defines a measure of non-homogeneity and is evaluated in the immediate neighborhood of $y_{s}$, its response could be handled as being a transformation that maps the gray-level of $y_{s}$ to the potential function $V_{c}\left(x_{s}\right)$. This relation is carried out in a proportional formulation as follows:

$$
V_{c}\left(x_{s}\right) \propto \tilde{g}\left(y_{s}\right)
$$

So, the MRF field henceforth be written in the following as:

$$
p\left(x_{s}\right)=\frac{1}{Z} e^{-\widetilde{g}\left(y_{s}\right)}
$$

\subsection{Level Set Speed Function of the Proposed Model}

We can integrate the boundary probability and region homogeneity information to define new speed function. The estimation of these parameters to find the boundary is posed as an optimization process, By applying the Bayesian formulation the objective function measures the strength of the data consistency term $h_{I}$ at a point $s$ is defined as decreasing function as following:

$$
p\left(x_{s} \mid y_{s}\right) \propto p\left(x_{s}\right) p\left(y_{s} \mid x_{s}\right)
$$

where $p\left(x_{s}\right)$ is the boundary probability term in (19), $p\left(y_{s} \mid x_{s}\right)$ is the object region probability term in (14). It is clear that (20) combines the statistical and the gradientbased measure in order to find the optimal segmentation. The new consistency term $h_{I}$ at point $s$ proportion to the combined probability $p\left(x_{s} \mid y_{s}\right)$ of the closest point on the current interface. The final expression of the new term $h_{I}$ will be:

$$
h_{I}(s)=\exp \left\{-k \cdot p\left(x_{s} \mid y_{s}\right)\right\}
$$

So the new speed function can be defined as:

$$
F=h_{I}(v-\rho k)=(v-\rho k) \cdot \exp \left\{-k \cdot p\left(x_{s} \mid y_{s}\right)\right\}
$$

\section{Experimental Results}

To demonstrate the performance of our new speed function for the level set framework, we carried out a series of experiments on medical images.

We describe a number of 2D images from which we extract the contours using the proposed method, which we have previously described. We have chosen images from some kinds of modalities medical images, US, CT, and X-ray images to demonstrate our methods. 
As we can see, the US image quality is very poor and the region boundaries seem to be very fuzzy. Fig.1 shows the results on a US image with both strong and fuzzy region boundaries. Fig.1 (a) shows the original image, Fig.1 (b) shows the initial curve, Fig.1(c) shows the Intermediate iterations process, Fig.1 (d) shows the result of the proposed method. The examples presented show that the algorithm we propose is able to cope with ultrasound images that are notoriously difficult to segment because of speckle noise as well as with images with low signal-to-noise ratio and poorly defined edges.

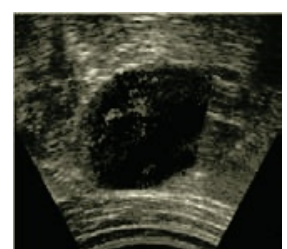

(a)

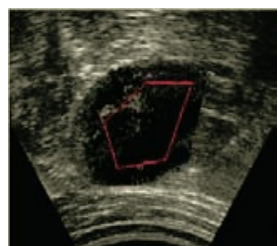

(b)

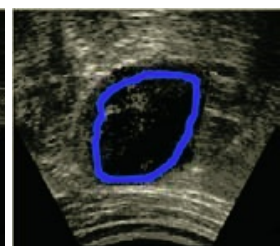

(c)

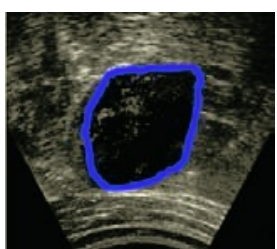

(d)

Fig. 1. US image segmentation results; (a)original image; (b) initial curve; (c) intermediate iterations; (d) our proposed method result.

We also applied our method on CT image of the liver as shown in Fig.2 and Fig.3. In every instance, a pair of image is presented. The human liver and brain images illustrate the performance of our methods for the segmentation of structures with similar intensity values that are separated by weak edges.

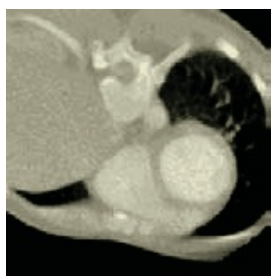

(a)

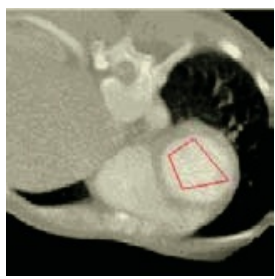

(b)

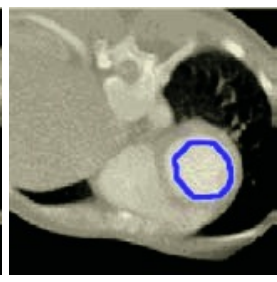

(c)

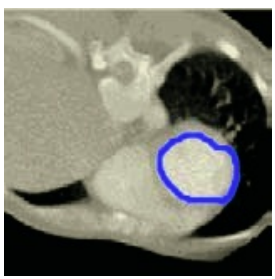

(d)

Fig. 2. CT liver image segmentation results; (a)original image; (b) initial curve; (c) intermediate iterations; (d) our proposed method results.

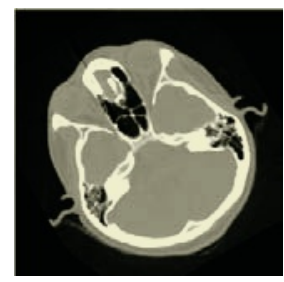

(a)

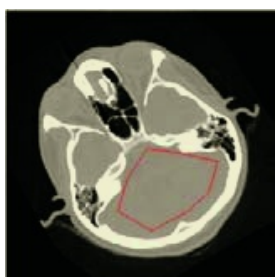

(b)

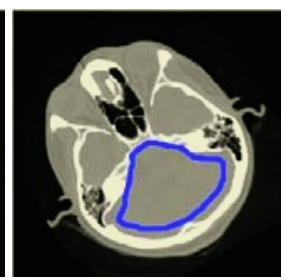

(c)

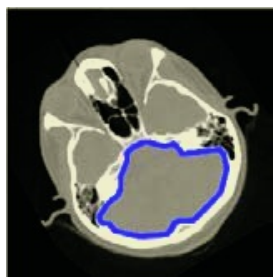

(d)

Fig. 3. CT brain image segmentation results; (a)original image; (b) initial curve; (c) intermediate iterations; (d) our proposed method results. 
Fig.4 shows the results of applying our methods for finding boundary of the X-ray carpal bone image with object overlap. This segmentation is a critical operation in the automatic skeletal age assessment system. The results clearly demonstrate the superior segmentation quality of our approach.

As mentioned above, the proposed method seems ideal for use on a wide variety of medical imagery. The power of this method in extracting feature from even fuzzy boundary and overlap boundary medical images has been demonstrated.

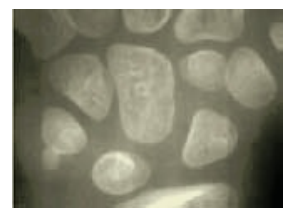

(a)

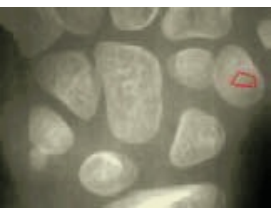

(b)

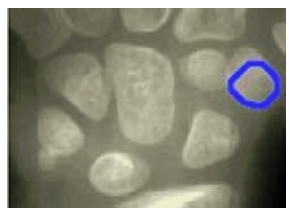

(c)

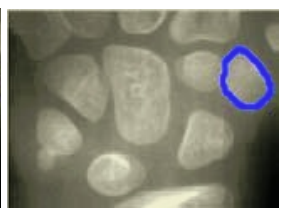

(d)

Fig. 4. Results of contour extracting from X-ray carpal bone image; (a)carpal bone; (b) initial curve; (c) intermediate iterations; (d) our proposed method result.

\section{Conclusions}

In this paper, we have proposed a new speed function for level set framework. The new models modify the level set speed function utilizing region intensity information and gradient information. The scheme here proposed is particularly well adapted to situations where edges are weak and overlap, and images are noisy. The method has been tested with numerical real modalities medical images, such as US, CT and X-ray images. The experimental results show the reliability of the approach.

\section{References}

1. S. J. Osher and J. A. Sethian. Fronts propagating with curvature dependent speed: Algorithms based on Hamilton-Jacobi formulations. J. Comput. Phys, vol. 79, pp. 12-49, 1988.

2. R. Malladi, J. Sethian, and B. Vemuri. Shape modeling with front propagation: A level set approach. IEEE T-PAMI, vol. 17, no. 2, pp. 158-175, 1995.

3. A. Chakraborty, L. Staib, and J. Duncan. Deformable boundary finding in medical image by integrating gradient and region information. IEEE T-MI, vol. 15, no. 6, pp. 859-870, 1996.

4. R. Ronfard. Region-based strategies for active contour models. International Journal of Computer Vision, vol. 13, no. 2, pp. 229-251, 1994.

5. C.S .Poon and M. Braun. Image segmentation by a deformable contour model incorporating region analysis. Phys. Med. Biol, vol. 42, no. 9, pp. 1833-1841, 1997.

6. C. Xu, A. Yezzi. Jr, and J. Prince. On the relationship between parametric and geometric active contours. Technical Report JHU/ECE 99-14, Dec. 1999.

7. C. Baillard and C. Barillot. Robust 3D segmentation of anatomical structures with level sets. Proceedings of MICCAI 2000, pp. 237-245, 2000.

8. H.Voorhees and T.Poggio. Detecting textons and texture boundaries in natural images. Proc.of the International Conference on Computer Vision, pp.250-258, 1987.

9. S. Geman and D. Geman. Stochastic relaxation, Gibbs distributions and the Bayesian restoration of images. IEEE Trans. on Pattern Analysis and Machine Intelligence, Vol.6, pp.721$741,1984$. 